\title{
Transcranial Doppler and angiographic findings in adolescent stretch syncope
}

\author{
Matthias Sturzenegger, David W Newell, Colleen M Douville, Sheila Byrd, \\ Ketti D Schoonover, Steve C Nicholls
}

\begin{abstract}
The clinical, angiographic, and Doppler findings are reported for two young patients with recurrent syncope induced by neck hyperextension during stretching. Transcranial Doppler monitoring of both posterior cerebral arteries was performed during head manoeuvres. There were reproducible rapid decreases in blood flow velocities in both patients to an average of $28 \%$ and $41 \%$ of baseline values when they performed neck hyperextension. These decreases were regularly associated with the patients' symptoms and were not found during rotation or flexion. On return to a neutral head position there was a transient increase of the blood flow velocities to an average of $131 \%$ and $136 \%$ of baseline values (reactive hyperaemia). Routine four vessel angiography was normal except for the demonstration of very small posterior communicating arteries in one patient. Dynamic angiography showed evidence of extracranial compression of the craniocervical arteries in both patients. It is concluded that decreased blood flow to the posterior circulation is an important factor in the pathogenesis of adolescent stretch syncope.
\end{abstract}

(F Neurol Neurosurg Psychiatry 1995;58:367-370)

Keywords: syncope; stretch; vertebral artery; Doppler

Department of

Neurological Surgery and Department of Surgery (SCN), Harborview Medical Center, University of Washington, Seattle, WA, USA

$M$ Sturzenegger

D W Newell

C M Douville

$S$ Byrd

K D Schoonover

$S$ C Nicholls

Correspondence to:

Dr M Sturzenegger,

Neurologische

Neurologische

Inselspital, CH-3010 Bern,

Received 17 May 1994

and in final revised form

6 October 1994.

Accepted 27 October 1994

\section{Case reports}

PATIENT 1

A 15 year old boy had been involved in a motor vehicle accident and sustained a whiplash type neck injury without radiological evidence of any lesion of the cervical spine. He subsequently noticed recurrent episodes of lightheadedness, dizziness, and blurred vision that eventually led to loss of consciousness when he kept his head maximally hyperextended and his arms flexed and hyperabducted (stretching). The symptoms resolved immediately when he flexed his head. The symptoms occurred most often when stretching in the sitting position. There were no other precipitating stimuli. Clinical examination was normal. Radiographs of the cervical spine showed incomplete segmentation failure of the $\mathrm{C} 3$ and $\mathrm{C} 4$ vertebral bodies. Magnetic resonance imaging studies of the head and upper cervical spine were normal. Doppler and duplex examination of the extracranial carotid and vertebral arteries and transcranial Doppler examination of basal intracranial arteries with the patient supine in a neutral head position were normal. Insonation of the P1 and P2 segments of both posterior cerebral arteries during common carotid artery compression did not show collateral flow through the posterior communicating arteries.

Simultaneous Doppler monitoring of both posterior cerebral arteries was performed with the patient in a sitting position using a two channel $2 \mathrm{MHz}$ Doppler system (Multi-Dop X/TCD7, Firma DWL, Elektronische Systeme GmbH, Sipplingen, Germany). The transcranial Doppler transducers were fixed to both temporal regions with a head band. The posterior cerebral arteries were identified on both sides according to standard criteria considering insonation angle, depth of sample volume, adjacent signals from the middle and anterior cerebral arteries, and flow direction of the signals with respect to varying depth of the sample volume. ${ }^{3}$ A transmission of pressure waves to the recorded posterior cerebral artery signals was seen on both sides with rapid recurrent suboccipital compression of either vertebral artery. While blood flow velocities in both posterior cerebral arteries were simultaneously monitored on line, the patient was instructed to perform the following head movements sequentially: rotation to 


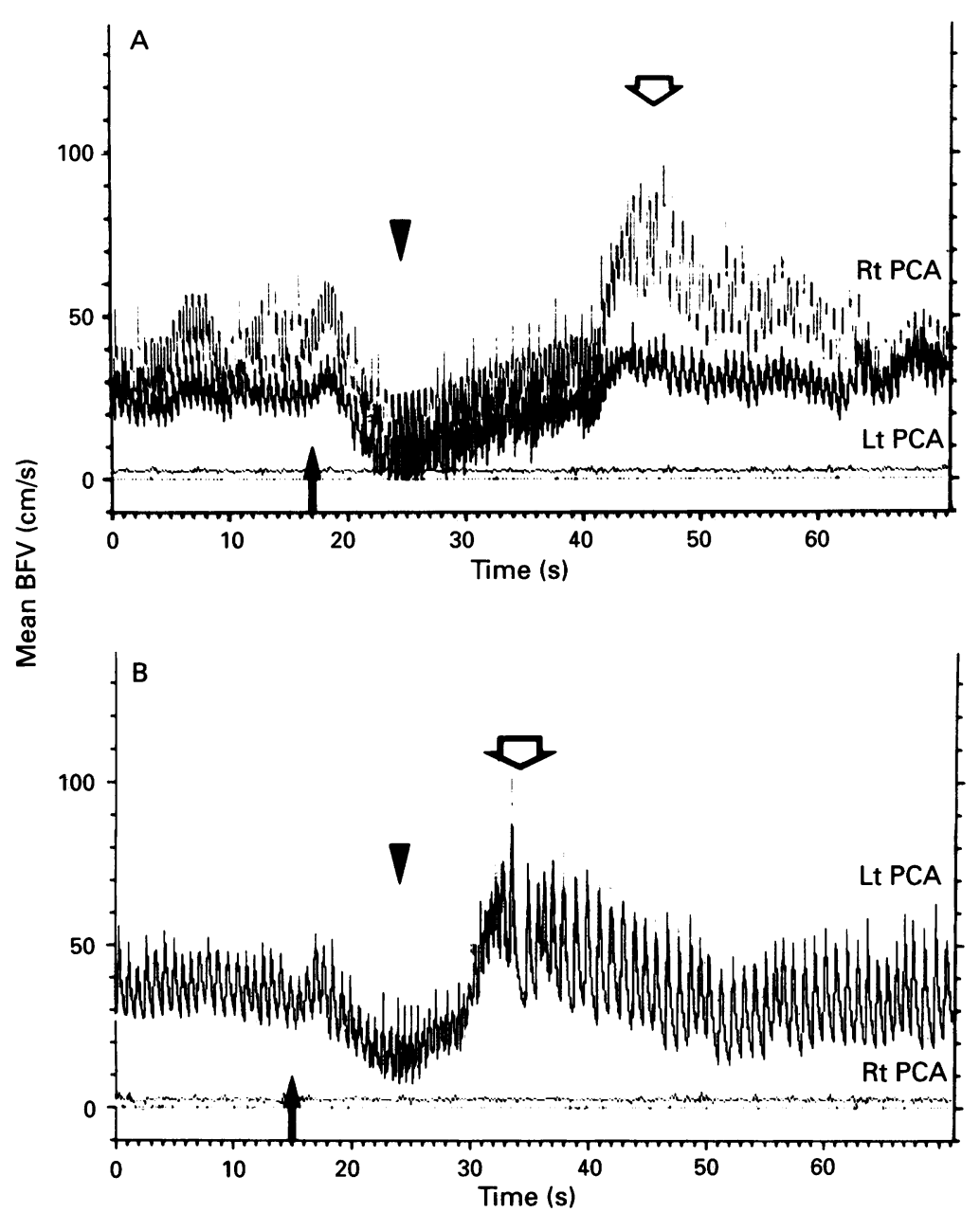

Figure 1 Time course of blood flow velocities (BFVs) (mean, maximal) in the left (lt) and right $(r t)$ posterior cerebral artery $(P C A)$ of patient $1(A)$ and patient $2(B)$. In $(B)$ the two traces almost exactly match. About three seconds after the start of stretching (arrow) there is a pronounced drop in BFV in both PCAs (arrowheads). This is followed by a transient increase above baseline values on returning the head to a neutral position (reactive hyperaemia; open arrow). either side, rotation with extension, maximal flexion, and maximal extension. These manoeuvres were also performed simultaneously with bilateral arm flexion and hyperabduction. During maximal head extension and arm hyperabduction there was a pronounced decrease in blood flow velocity with dampened pulse waves in both posterior cerebral arteries (fig 1A). The patient complained of dizziness and blurring of vision about four seconds after the decrease in blood flow velocity. When he moved his head back to the neutral position, there was a transient hyperaemic response indicated by blood flow velocities above baseline values (fig 1A). Similar changes in blood flow velocity in the posterior cerebral arteries could be induced several times in this position. The mean blood flow velocity during four trials of hyperextension dropped to a minimum of $12 \%$ of the baseline value (mean 28 (SD 10.3)\%; range $12-44 \%)$. Postischaemic hyperaemia caused a transient increase of the mean blood flow velocity to a maximum of $169 \%$ of the baseline value (mean 131 (SD 19.1)\%; range $107-169 \%$ ). Maximal rotation or flexion of the head, however, caused no significant changes in blood flow velocity. The patient subsequently had four vessel catheter angiography in a neutral position and with dynamic manoeuvres. Both carotid and vertebral arteries in the neutral position were normal. Both posterior communicating arteries, however, were very small. The attempt to image vertebral artery compression was only partially successful as the patient was unable to reproduce the position during the arteriogram, which made him prone to syncope. During hyperextension and arm hyperabduction, however, a moderate amount of narrowing was produced in the right proximal vertebral artery (V1-V2 junction).

\section{PATIENT 2}

This 18 year old man had recurrent syncopal episodes triggered by neck hyperextension and abduction and external rotation of the shoulders (stretching). Most of the syncopal episodes were preceded by dizziness and a dull occipital headache. Other head movements did not precipitate such spells. He was otherwise healthy and detailed clinical examination, conventional cervical spine radiographs and MRI studies of the head and upper cervical spine were normal. Doppler and duplex examination of the extracranial carotid and vertebral arteries and transcranial Doppler examination with the patient supine with a neutral head position were normal. Transcranial Doppler monitoring of the basilar artery with a suboccipital approach was performed during the provocative position, which showed reduced blood flow velocity and a dampened flow velocity profile shortly before the patient fainted. Interpretation was difficult, however, due to probe motion artefacts.

Transcranial Doppler monitoring of both posterior cerebral arteries simultaneously by a transtemporal approach during different head movements was performed with the same technique and protocol described for patient 1. Monitoring the P1 segment showed a significant decrease in blood flow velocity in both posterior cerebral arteries only during maximal hyperextension (fig 1B). The other head positions or positioning of the arms only without hyperextension of the head did not induce significant changes in blood flow velocity. Again, these decreases could be induced repetitively and the patient consistently reported his symptoms. When his head was turned back to a neutral position, a short transient episode of reactive hyperaemia was seen. The maximal drop recorded during four trials of hyperextension was to $20 \%$ of the baseline value (mean 41 (SD 10.4)\%; range $20-54 \%$ ). Maximal postposition increase in blood flow velocity was to $203 \%$ of the baseline value (mean 136 (SD 30.8)\%; range 105-203\%). Routine four vessel angiography with the head in a neutral position demonstrated normal carotid and vertebral arteries 

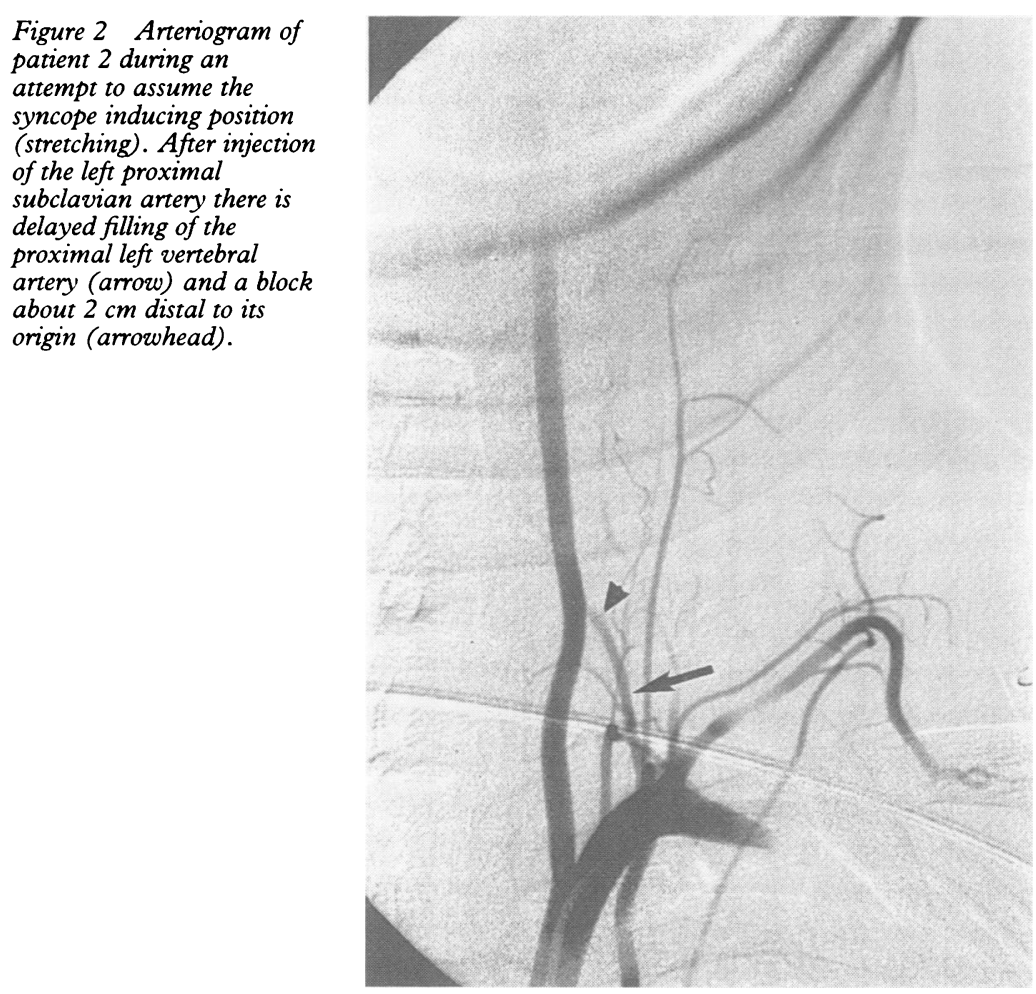

bilaterally and normally sized posterior communicating arteries. A second angiogram performed with the patient in the syncope inducing position showed delayed filling of the left vertebral artery with opacification only of the most proximal $2 \mathrm{~cm}$ (fig 2) whereas there was good filling of the right vertebral artery with filling into the basilar artery and both postenior cerebral arteries. A right carotit arteriogram was normal, whereas the left internal carotid artery was faintly opacified up to the skull base but not beyond. The examination then had to be terminated before completion as the patient lost consciousness and then became combative with a state of disorientation that lasted about 30 minutes. Computed tomography with contrast enhancement was normal.

\section{Discussion}

The crucial position to induce stretch syncope is hyperextension of the head and neck. In our patients and others reported in the medical literature, syncope occurred only in the sitting or standing position but not supine, which may indicate incomplete neck hyperextension when stretching supine due to head contact with the surface rather than orthostasis. These patients usually do not have a history of syncope other than that associated with neck hyperextension. Stretching alone without associated neck hyperextension in our two patients did not induce syncopal symptoms or a decrease in blood flow velocity. Thus Valsalva's manoeuvre cannot be the (only) explanation although studies have shown that this manoeuvre is performed while stretching. ${ }^{2}$
Stretch syncope does not have an established aetiology. Aura symptoms preceding loss of consciousness, such as vertigo, visual blurring, tinnitus, and numbness in the extremities are typical of vertebrobasilar ischaemia and their strict association with neck hyperextension favours vertebral artery compression. ${ }^{4}$ One can make an argument for mechanical extrinsic vertebral artery compression from its occurrence predominantly in adolescents, as it is well known that the physiological ranges of all movements in the cervical spine and craniocervical junction decrease with age. ${ }^{5}$ It has been established in arteriographic studies performed on cadavers and volunteers as well, that head rotation and extension can cause contralateral, ipsilateral, and more rarely, bilateral vertebral artery obstruction, most likely in the mobile atlantoaxial segment. ${ }^{67}$ Minor anomalies of the craniovertebral junction, such as condylar hypoplasia, ${ }^{8}$ may lead to some degree of occipitoatlantal instability with the risk of vertebral artery compression. ${ }^{59}$ Such anomalies may be difficult to verify and require special diagnostic techniques. ${ }^{10}$ Patient 1 had a vertebral body fusion, which is often associated with dysplasia of the craniocervical junction. ${ }^{9}$ Studies with MRI did not show abnormalities of the posterior fossa or craniocervical junction.

The posterior circulation has special anatomical characteristics that enable multiple collateral channels at several levels hetween the origin of the vertebral arteries and the posterior cerebral arteries to preserve cerebral blood flow. If one vertebral artery is occluded, there are two primary collaieral pathways working immediately: the opposite vertebral artery and the circle of Willis with the posterior communicating arteries. Thus it would normally require high grade bilateral vertebral artery obstruction or unilateral obstruction in the presence of contralateral vertebral artery anomaly (hypoplasia, termination in the posterior inferior cerebellar artery), or disease (stenosis, thrombosis) to cause haemodynamic ischaemic symptoms. This, however, is not necessarily sufficient, as it is known that even bilateral vertebral artery disease may be compensated for by efficient collateral flow in the posterior communicating arteries. Patient 1, who showed normal anatomy of the vertebrobasilar system on angiography, but very small and thus haemodynamically inefficient posterior communicating arteries, illustrates the importance of poor collateral flow from the anterior circulation. It is, however, difficult to assess their haemodynamic efficiency from the angiographic appearance of the posterior communicating arteries in the case of bilateral vertebral occlusion. Patient 2 had normally sized posterior communicating arteries. His inefficient collateral supply from the anterior circulation is probably due to simultaneous haemodynamic effective obstruction of the left internal carotid artery during maximal hyperextension of the neck. It is well known that the internal carotid artery may be 
stretched over the transverse process of the atlas during forced head extension. ${ }^{611}$

The described method of transcranial Doppler monitoring of blood flow velocities of posterior cerebral arteries may have advantages compared with angiography, as various combinations of head movements can be performed sequentially and analysed for their influence. The high time resolution of transcranial Doppler is particularly useful to show rapid and brief haemodynamic changes. This approach measures the net haemodynamic effect of possible obstructions at different levels and is therefore likely to correlate with clinical symptoms. The absence of a so called temporal bone "ultrasound window" may preclude investigation of posterior cerebral arteries in a considerable percentage of elderly people. This is not usually a problem when examining adolescents, the population at risk for stretch syncope. The technique reported here of monitoring both posterior cerebral arteries by a temporal approach using a two channel Doppler system as compared with basilar artery and vertebral artery monitoring by a suboccipital transforamenal approach with the freehand technique has the advantage of stable Doppler signals despite head movements. ${ }^{12}$ Suboccipital transducer position is hampered by large probe motion artefacts during head movements as well as the risk of signal loss due to changing depth of the vessel of interest and difficulties maintaining vessel insonation. ${ }^{13}$

The natients described here show that it may be difficult to prôvie vertebräal artery compression during angiography because of the inability to reproduce thi exact offending posture. Also, angiography is invasive and not without risk. Transcranial Doppler monitoring on the other hand, although able to prove haemodynamically significant vertebral artery compression, does not identify its level and the underlying cause. ${ }^{14}$ Avoidance of the triggering posture in most patients is all that is necessary to eliminate further attacks.

1 Manolis AS, Linzer M, Salem D, Estes MNA. Syncope: current diagnostic evaluation and management. Ann Intern Med 1990;112:850-63.

2 Pelekanos JT, Dooley JM, Camfield PR, Finley J. Stretch syncope in adolescence. Neurology 1990;40:705-7.

3 Buedingen HJ, Staudacher T. Evaluation of vertebrobasilar disease. In: Newell DW, Aaslid R, eds. Transcranial lar disease. In: Newell DW, Aaslid R, eds. Transc

$4 \mathrm{Grad}$ A, Baloh R. Vertigo of vascular origin. Arch Neurol 1989;46:281-4.

5 Gilles FH, Bina M, Sotrel A. Infantile atlanto-occipital instability: the potential danger of extreme extension. Am $₹$ Dis Child 1979;133:30-7.

6 Toole JF, Tucker SH. Influence of head position upon cerebral circulation. Study of blood flow on cadavers. Arch Neurol 1960;2:616-23.

7 Faris AA, Poser CM, Wilmore DW, Agnew CH. Radiologic visualization of neck vessels in healthy men. Neurology 1963;13:386-96.

8 Schmidt $\mathrm{H}$, Sartor K, Heckel RW. Bone malformations of the craniocervical region. In: Vinken PJ, Bruyn GW, the craniocervical region. In: Vinken PJ, Bruyn GW, North-Holland Publishing Co, 1978:1-98.

9 Coria F, Rebollo M, Quintana F, Polo JM, Berciano J. Occipitoatlantal instability and vertebrobasilar ischemia Case report. Neurology 1982;32:303-5.

10 Wiesel SW, Rothman RH. Occipitoatlantal hypermobility Spine 1979;4:187-91

11 Saver JL, Easton JD, Hart RG. Dissections and trauma of cervicocerebral arteries. In: Barnett HJM, ed. Stroke. Pathophysiology, diagnosis and management 2 nd ed. New York: Churchill Livingstone, 1992:671-88.

12 Brautaset NJ. Provokable bilateral vertebral artery compression diagnosed with transcranial Doppler. Stroke pression diagnos

13 Fujioka KA, Ernsberger AM, Nicholls SC, Spencer MP. Transcranial Doppler assessment of mechanical compression of the vertebral arteries. Fournal of Vascula Technology 1991;15:254-9.

14 George B, Laurian C. Impairment of vertebral artery flow caused by extrinsic lesions. Neurosurgery 1989;24 206-14

Marcel Proust, 1923, 1925, 1927, Rememberance of things past. Vol 3

"I was very distressed when I was told that he had lost his sight." "Yes, it's true that his eyes were affected by his stroke. For a time he could see nothing at all, jus imagine, during the cure, which as a matter of fact did him a great deal of good, he was for several months unable to see more than a man born blind."

Gaius Suetonius (AD69-140), The twelve Caesars translated by Robert Graves, 1957

In reference to Nero

A warning portent made him cancel the Alexandrian voyage, on the very day when his ship should have sailed: during his farewell round of the temples he had sat down in the shrine of Vesta, but when he rose to leave, the hem of his gown caught fire and then a temporary blindness overcame him.

G D PERKIN Regional Neurosciences Centre Charing Cross Hospital, London W6 8RF, UK 